\title{
Living with Advanced Hormone-Sensitive Prostate Cancer and Treatment with Abiraterone and Androgen Deprivation Therapy: The Patient, Nursing and Physician Perspective
}

\author{
Tony Collier · Shievon Smith • Michelle Greenwood · Kenrick Ng (D)
}

Received: September 24, 2020 / Accepted: September 30, 2020 / Published online: October 9, 2020

(c) The Author(s) 2020

\begin{abstract}
This article is co-authored by a patient with metastatic hormone-sensitive prostate cancer who is receiving abiraterone and androgen deprivation therapy treatment in Manchester, UK. The patient relates his personal experiences struggling with the diagnosis, his experience with treatment and the physical, emotional and psychosexual impact on his life. After his diagnosis, the patient has become an outspoken advocate and fundraiser for prostate cancer awareness and wants to ensure that novel treatments with proven efficacy and tolerability, such as abiraterone, are available for all men in his condition. The specialist nursing and
\end{abstract}

T. Collier

Prostate Cancer UK, 53 Tooley Street, London SE1

2QN, UK

S. Smith · M. Greenwood · K. Ng

St Bartholomew's Hospital, Barts Health NHS Trust, London EC1A 7BE, UK

K. Ng (ه)

UCL Cancer Institute, 72 Huntley Street, London

WC1E 6DD, UK

e-mail: k.ng@ucl.ac.uk physician perspectives, provided by healthcare professionals based in London who are not directly involved in this patient's care, were written in response to the challenges and concerns highlighted by this patient. The role of the specialist nurse as a key healthcare professional in the cancer patient journey, particularly in managing the complex physical and emotional side effects of treatment, is highlighted in this perspective piece. The physician reviews the current difficulties of establishing an effective screening programme in prostate cancer, the common side effects of hormone treatment and the significant progress and challenges in novel drug development and prescription in metastatic hormone-sensitive prostate cancer. While written primarily from the perspective of a patient and healthcare professionals in England, many messages in this commentary would resonate with patients and professionals involved in the care of prostate cancer worldwide.

Keywords: Abiraterone; Patient perspective; Prostate cancer; PSA; Screening; Survivorship 


\section{Key Summary Points}

This article is co-authored by a patient with advanced prostate cancer on abiraterone and androgen deprivation therapy.

It details his struggles with the initial diagnosis, his experiences with treatment, the impact this has had on his personal and family life and his role as a patient advocate.

The perspective of clinical nurse specialists is also provided, to highlight the important role of these professionals in managing the complex physical and emotional side effects of treatment in the patient journey.

The physician responds to concerns and queries relating to screening programmes, side effects of treatment and challenges relating to treatment availability and reimbursement.

\section{DIGITAL FEATURES}

This article is published with digital features, including a summary slide, to facilitate understanding of the article. To view digital features for this article go to https://doi.org/10.6084/ m9.figshare.13020092.

\section{MY STORY}

I was diagnosed in May 2017, aged just 60, with a prostate-specific antigen (PSA) level of 129, Gleason $5+4$ with metastases pretty much throughout my skeleton from pelvis to skull. I was completely asymptomatic until February 2017 when I started with a niggling runners' groin strain whilst training to take part in an ultra-marathon in South Africa in early June 2017. The groin strain worsened so much that I had it checked by a sports injuries doctor and

we pre-arranged a MRI (magnetic resonance imaging) scan. When I was called into the doctors' office I was told that the scan was not showing what they expected and that I would need further tests to rule out anything untoward. He sent me there and then for chest X-rays and blood tests; the first time I saw the handwritten letters "PSA" and I had no idea what that meant. I had a CT (computed tomography) scan scheduled for the next day. The night before the scan was a very sleepless one as clearly you do not need those tests for a groin strain. The day of the CT scan the doctor called me at $8.00 \mathrm{pm}$ to tell me that I almost certainly had prostate cancer but would need further tests to confirm the position. He dealt with this by telephone as he wanted to speed these tests through, but it was a real shock. I drove home shaking and in floods of tears to break the news to my wife. The first thing I did was to push her away by telling her she should go and find someone else because I was no longer any use to her and would not be able to look after her any more. Little did I know then how much my diagnosis would impact on her life and how important it is to have a strong relationship to help get through the difficult days, months and years ahead.

Ten days of further tests confirmed that initial diagnosis, and my urologist told me that I may only have 2 years to live. He also suggested that I probably had the prostate cancer for 10 years, totally asymptomatic. It turns out that the groin strain was stress fractures of the pelvis where the cancer had eaten into the pelvic bone. It is staggering to think that I had run 20 marathons and an ultra-marathon all whilst this cancer was growing inside me (Fig. 1).

I met my oncologist on 31 May 2017 for the first time. By the date of that first appointment I had been taking bicalutamide for 2 weeks and had had my first leuprorelin injection.

The oncologist proposed docetaxel chemotherapy commencing in August 2017 and I was all set for that. Plenty of false bravado with a "Bring it on" attitude when deep down I was terrified of the impact that chemotherapy might have on me.

I was very open about my diagnosis once I had got past the worst experience of my life: 


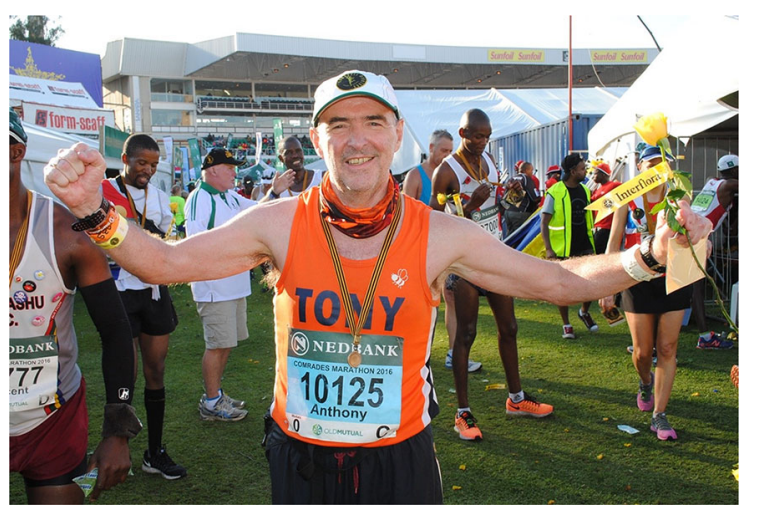

Fig. 1 The joy of having completed a 56-mile ultramarathon, 12 months pre-diagnosis

telling my adult children. Thankfully they live within $30 \mathrm{~min}$ of us, but I had to ask them to come round one evening because I had some news that I needed to tell them. Goodness knows what they imagined was going on, but I can pretty much guarantee that it was far worse than their worst fears.

Due to being so open about my illness a number of friends around the world contacted me in June 2017 to tell me about this new 'wonder drug', abiraterone. They had heard about the announcement of the efficacy of abiraterone as first line treatment at the ASCO (American Society of Clinical Oncology) 2017 annual meeting. I thought it would be worth discussing with my oncologist, and he felt that it would be very effective for me and managed to persuade my private medical insurers to fund it for me.

I started on abiraterone in early July 2017 but initially had some liver function issues. This is a known side effect of abiraterone for some men and means that men prescribed the drug need regular blood tests to monitor liver function. I am told that the normal range of AST (aspartate aminotransferase) liver function is 10-40 units/ L but mine just kept on going up and up. This meant coming off the drug for 6 weeks and then trying again. This cycle was repeated a number of times and, ultimately, by reducing the dosage to $500 \mathrm{mg}$ daily from the standard $1000 \mathrm{mg}$, the liver function finally settled but it had been over 300 units/L at one stage. At one point my oncologist said I should come off the drug and I begged for another 3 weeks. Amazingly, in that 3 weeks, things stabilised and my liver function has remained at the high end of the normal range ever since.

There was a funny story from one of my visits to see the oncologist when my wife asked "Given Tony's liver function problem is his alcohol intake an issue?" My doctor's response was classic "I'm from Glasgow, what do you expect me to say?" before turning to me and saying "Carry on as before".

The other thing I like about my oncologist is that he's a sportsman and really understood what my running meant to me. When I asked would I still be able to run he said that once the stress fractures had healed I would be able to resume training and, in fact, actively encouraged me to do so. I have since learnt that not all oncologists are tuned in to how exercise can help physical and mental wellbeing! He did, however, very carefully manage my expectations by talking me through the side effects of being on hormone therapy and informing me that loss of muscle mass, reduced bone density, weight gain and fatigue would result in me being a lot slower and I had also struggle to maintain endurance. He was of course correct and it has been one of the things I've struggled with most since my diagnosis.

Most importantly after the initial diagnosis and discussions my PSA level fell dramatically with the combination of leuprorelin and abiraterone and reached an unrecordable level within 10 months and has stayed there ever since, now over 3 years post diagnosis in July 2020 .

\section{Mental Impact}

Being told that you have cancer is horrendous; being told that it is incurable is indescribable. At the time my grandson was only 3 years old and I utterly doted on him. I had many dark thoughts and cried myself to sleep many times thinking that I probably wouldn't get to see him become a teenager. I was directed to the Maggie's centre (an organisation that provides support to cancer patients and their families), which has been a godsend. I was also very lucky to have a 
wonderful clinical nurse specialist (CNS) who was also massively supportive and reassuring. I think that the role of CNS is so vital in cancer care and all cancer patients should be supported by one although I know that that is not always the case.

Four really important messages flow from my experience of the initial dark days. Firstly, you feel so much better when you start off on a treatment pathway. Secondly, as time passes things become much easier. Thirdly, it is likely that you'll have a meltdown from time to time but you do learn to accept that it is okay not to be okay every now and then. Finally, if you do get emotionally overcome there is support out there and it's a sign of strength to seek out help. I had a massive meltdown in October 2018. I sought help from the Neil Cliffe Centre and spent $2.5 \mathrm{~h}$ in floods of tears with a case worker. I did not feel weak for asking for help!

\section{Side Effects}

The oncologist was not wrong about running getting tougher. At 60, pre-diagnosis, I was running $5 \mathrm{~km}$ in $22 \mathrm{~min}$. Now a really good day is $26 \mathrm{~min}$ and $\mathrm{I}$ have to admit that it ripped me apart. Gradually though, I have grown to accept it, begrudgingly, and I still run regularly. As a massive advocate of exercise I try hard to walk the walk. I think it is really important to maintain physical and mental wellbeing and I am convinced it will extend my prognosis. I think there is a really important message here for people living with cancer and coping with side effects like me. We can still do lots of things if we do not let our mind stop us from doing so. Positivity is vital.

The other side effects that impact on men on hormone therapy are loss of libido and erectile dysfunction. Basically, sex never crosses your mind and you have no enthusiasm to even engage in intimacy. In fact I know that there have been times, still are, where I have shied away from physical contact with my wife because I was scared it would lead to sex and I wouldn't be able to get an erection.

This emphasises the point I made that a prostate cancer diagnosis affects relationships but we are in this together and we try hard to make things work.

It also reminds me of a conversation with my oncologist when he explained the impact of hormone therapy by saying "Regrettably, once you start on this treatment you'll be unable to get an erection but it probably won't matter as you'll not want one due to loss of libido". When I asked if there was any other bad news to come he said "Yes, you'll experience penile shrinkage!" Of course this is a total embellishment of that conversation but it's also indicative of how my wife and I try to deal with the issue with humour. In fact the other day she asked me to put some music on so I asked her what she'd like and she said "I'd love a bit of sax". Well, I nearly crapped myself.

Joking apart, it is a serious problem. I have been lucky again to be able to get support from another CNS who is also a psychosexual therapist and she explained that hormone therapy takes away your drive but it doesn't take away your desire and we have focussed on those words to help us maintain intimacy. Regrettably I will never have a strong enough erection to have penetrative sex again, even with chemical support, but it does not stop us enjoying each other's bodies and when I do manage to reach orgasm it is of totally mind blowing intensity and, by using sex toys we have learnt how to pleasure each other. It's not the same but it is better than the alternative.

One thing that I think men can hope for in the future is the development of a treatment that does not completely emasculate them. Is that ever likely?

\section{Life Changes}

It would be fair to say that no aspect of life has remained the same since my diagnosis. They call it the new normal but I prefer the new abnormal as nothing will ever be normal again. With a worst case prognosis of 2 years and an average of 6-7 years it really focusses your mind and you think about priorities. Mine is to live life to the full while I am still fit and well.

I think that there is a salutary lesson here that people shouldn't wait for a cancer 
diagnosis to live life to the full. It may be too late when that comes along so do not put off until tomorrow those things that you really want to do today.

I decided to rid myself of anything causing stress. Dealing with cancer is hard enough without stress in your life!

One area of stress that you cannot avoid is blood anxiety, namely the 3-monthly PSA blood tests, but, again, it gets easier with the passage of time although I have no idea how I will react when I eventually get bad news, which I know I will.

\section{PSA Testing}

I knew nothing about prostate cancer before I was diagnosed but you very soon gather lots of knowledge. I was massively shocked to find out that I could have requested a PSA test from age 50 and then really angry that if I had asked for one every year from age 50 I could have been diagnosed much earlier and had curative treatment. Men really need to know about this which is why I am very vocal about my own diagnosis so that men get to know what happens if you do not get early diagnosis. A year after my diagnosis I managed to get a copy of my scan which shows the extent of the spread of my cancer and this really shocks people. My message is very clear. If you do not want to be
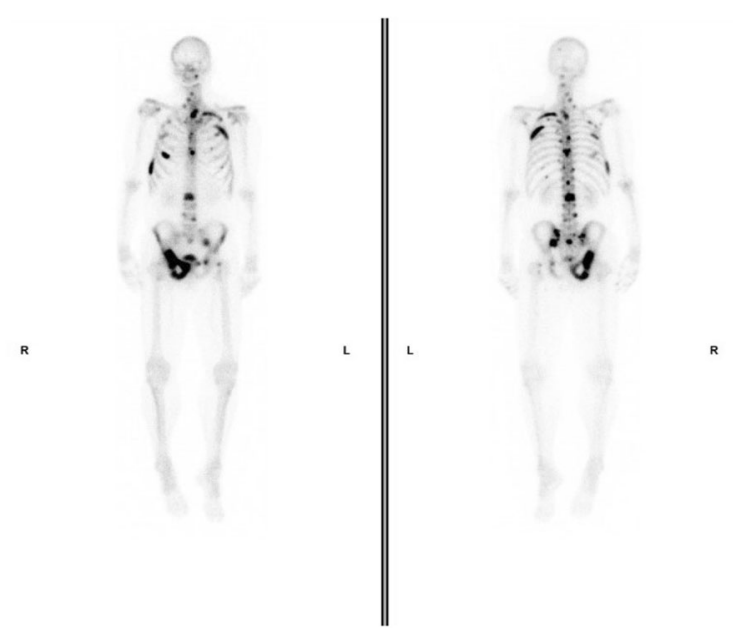

Fig. 2 Bone scan showing multiple bony deposits from metastatic prostate cancer like me, do something proactive about your prostate health (Fig. 2).

Regrettably many more lives will be lost as a result of late diagnosis until such time that we have an accurate tool to use for population screening and some general practitioners (GPs) stop refusing PSA tests for asymptomatic men. I hope that this won't be too far into the future and would like to know if we are any nearer to that tool being available and to what extent COVID-19 (coronavirus disease 2019) has slowed research into producing that diagnostic tool.

\section{Abiraterone Versus Docetaxel}

As already outlined I was originally scheduled to start treatment with docetaxel chemotherapy when I heard about abiraterone. Three years down the line I'm elated at how well abiraterone has worked for me but now I face a real problem with NICE (UK National Institute for Health and Care Excellence) having refused to allow abiraterone as first-line treatment in England and Wales.

I strongly believe that men diagnosed with an illness that is effectively life shortening should have choices available to them that enables them to maintain the best possible quality of life for what life they have left. I believe that docetaxel impairs short-term quality of life for a significant proportion of men and that for some men it permanently impairs their lives-and I have gathered plenty of evidence to support that position. However, NICE does not appear to be persuaded on that point. Thankfully they have agreed that there are grounds for our appeal.

In my own case, as a sub-elite athlete, docetaxel could have permanently impacted my life as a runner negatively whilst abiraterone enabled me to lead a full and active life virtually immediately post diagnosis. When I had the opportunity to get treatment with abiraterone I jumped at the chance.

The most appalling aspect of NICE's decision was to conclude that the manufacturer's commercial proposal on the price of abiraterone could not be used to inform the Committee's 
decision-making because that price proposal had not been approved by NHS (National Health Service) England. I find that absolutely disgraceful! Men's lives matter!

In my opinion, when considering the cost of docetaxel versus abiraterone a conclusion cannot be reached just on the cost of the drugs alone. Delivery of docetaxel entails hours in a hospital and is quite manpower intensive, requiring nursing oversight throughout. Further costs are incurred to deal with side effects when people have an adverse reaction to docetaxel.

Now I face a real dilemma as I cannot afford my health insurance premiums any more. We should not be faced with living with the worry of how on earth we are going to maintain supply of the drug that is keeping us alive on top of the mental challenge of living day-to-day with an incurable, effectively terminal, illness.

\section{NURSING AND PHYSICIAN PERSPECTIVE}

\section{Screening for Prostate Cancer-Current Landscape and Future Perspectives}

In the UK, approximately one in eight men will be diagnosed with prostate cancer in their lifetime. The risk factors for developing prostate cancer include age (above 50 years), family history and black ethnicity. Fortunately, most cases present at an early stage and often have an indolent course, requiring minimal or no treatment. The relative survival rate of patients with prostate cancer 10 years after diagnosis is over $98 \%$, because more than eight in ten patients are detected and treated early, giving them a good chance of cure [1]. Unfortunately, this favourable survival rate is not shared by the $10 \%$ of patients who are diagnosed at an advanced stage of cancer.

If the chances of cure are higher the earlier the prostate cancer is detected, why is there a conspicuous absence of a population-wide screening programme? There are a few reasons, with the key issue being the lack of a diagnostic test which is both sensitive and specific. The PSA is the best available blood test for prostate cancer but has its limitations, particularly its lack of specificity. Secondly, an overreliance on PSA may cause diagnosis of clinically indolent prostate cancer, leading to an unnecessary series of biopsies and treatments with side effects but no benefit to morbidity or mortality.

However, like with the case of our patient, the absence of a screening programme means some men with advanced prostate cancer slip through the net. While many men present with lower urinary tract symptoms, many others do not develop symptoms until the cancer has spread to other organs. In developing screening programmes, we frequently assess the benefit from a population level. However, we should bear in mind that while a screening programme may not produce overall survival benefit to a population, some patients may derive significant benefit from having their cancers detected early through screening. The key is in identifying and optimising a strategy for risk stratification for this cohort of patients.

In the UK, there is an informed choice strategy referred to as the Prostate Cancer Risk Management Programme. This programme guides GPs to counsel asymptomatic men aged $\geq 50$ years about the merits and harms of PSA testing, but only for those who proactively ask about testing. The Programme empowers the patient with the knowledge of the benefits of having their PSA checked, while acknowledging that this is an imperfect test which may lead to a series of unnecessary investigations and possibly treatments with no overall effect on his life expectancy.

Most patients and healthcare professionals would agree that an effective screening programme for this common cancer is desirable, if tests (or a combination of tests) had good predictive value. Several other tests are being studied to help detect clinically significant prostate cancer. A study into a panel containing four kallikrein proteins (total PSA, free PSA, intact PSA and human kallikrein) has demonstrated an ability to discriminate between men with indolent and aggressive disease [2]. Advanced imaging techniques, such as multiparametric MRI, have also been used as a triage test to avoid unnecessary biopsy and improve diagnostic accuracy [3]. Using a multimodality prediction model, our Swedish colleagues have 
developed a predefined model consisting of plasma protein biomarkers, genetic polymorphisms and clinical variables along with PSA to help diagnose high-risk prostate cancer [4]. While these are interesting scientific models, translating their results into clinical practice will involve validating these studies in different population settings and ensuring that individual healthcare systems have the technical expertise and resources to conduct these tests at a large scale for screening.

\section{Androgen Deprivation Therapy-The Backbone of Treatment of Hormone- Sensitive Prostate Cancer}

In 1941, Charles Huggins and Clarence Hodges published their findings that metastatic deposits from prostate cancer were hormonally sensitive [5]. Nearly 80 years after the publication of the classic Cancer Research article, androgen deprivation therapy (ADT) remains frontline treatment for advanced prostate cancer. The premise remains that, in most cases, testosterone and other androgens drive the growth of prostate cancer and that suppression of these hormones should inhibit the growth and potential of metastasis.

However, the modulation of testosterone levels can cause a range of side effects that negatively impact quality of life. It can cause vasomotor symptoms, resulting in hot flushes and associated sleep problems, as well as changes in body composition, with loss of lean body mass and increased body fat. ADT is also associated with changes in bone mineral density and has been associated with cardiovascular complications.

Perhaps the most significant and least openly discussed impact of ADT is its effect on sexual dysfunction. Many men receiving continuous ADT develop a degree of sexual dysfunction. Loss of libido usually develops in the first few months, and erectile dysfunction then follows. The poignant description offered by this patient, particularly the negative impact of erectile dysfunction on his sense of masculinity and sexual relationship, echoes an opinion shared by many in our patient population.
The last few years have heralded a transformation in the strategies we employ to treat prostate cancer. There is an increased drive to focus on precision medicine instead of the traditional paradigm of castrate-sensitive and castrate-resistant disease. For instance, the US FDA (Food and Drug Administration) approved rucaparib and olaparib for BRCA-mutant metastatic castration-resistant prostate cancer earlier this year. This represents a major change in the prostate cancer space, as this patient population had previously no biomarker-driven therapies. There is also increased focus on major signaling pathways, such as PI3K/AKT/mTOR, identified in between 70 and $100 \%$ patients with advanced prostate cancer. Clinical trials are now focussed on developing therapies towards components of these pathways [6].

These are novel developments in the landscape of treatment for prostate cancer. However, considering the relative efficacy of androgen deprivation as a treatment option, research in precision medicine will currently be focussed on the more advanced 'castrate-resistant stage' of the disease, where there is a greater unmet need. At this point, it is difficult to envision a world where we treat advanced prostate cancer without hormone manipulation in the first-line setting. However, as our understanding of the biology of prostate cancer continues to grow, we are hopeful that science will eventually produce an effective therapy based on targeted precision medicine.

\section{The Perspective and Role of Clinical Nurse Specialists}

Due to advances in scanning modalities and patient awareness, an increasing number of younger men are being diagnosed with prostate cancer, and often with de novo metastatic disease. Often, these men are working, might be starting families later in life or have a young family [7]. The diagnosis is emotionally laden to receive and, in turn, to share with family and friends. It is considered best practice for an oncologist to deliver bad news in clinic in the presence of a CNS who will provide important psychological assessment and support, and act 
as a keyworker with the relevant expertise and experience to address the patients' concerns, both during and after the consultation.

At this time, a holistic needs assessment would be completed, ideally alongside the patient's relative or carer. This assessment includes social circumstances, such as financial and family/friend support, a complex emotional assessment, lower urinary tract assessment, sexual function assessment and medication toxicity information, such as hot flushes or side effects from chemotherapy [8]. During this assessment, the explanation of the role of a CNS, management of patient's expectations and the provision of keyworker contacts for further support and oncology by out-of-hours hotline are also provided. Currently, during the COVID-19 pandemic visitors have been restricted, which often adds to the emotional burden of a patient when bad news is delivered. In these times, the role of the CNS for support is more important than ever, particularly in bridging communication between clinic, patient and their family.

This keyworker role remains one of the most gratifying elements of the job description for a CNS, but due to increasing demands the service is under strain. Research has highlighted that uro-oncology CNSs cover all five tumour sites and, on some occasions, provide benign services, such as continence assessments. This study confirmed that many uro-oncology CNSs carry caseloads of over 600 patients and that work can often be left uncompleted, or at least not carried out according to the standard desired by the nursing professional [9].

It is recommended that patients are active to aid recovery from treatments, long-term bone and cardiac health,and psychological health [10]. Metastatic prostate cancer patients are treated with long-term androgen deprivation and steroid manipulation, and these therapies come at a cost. As discussed, weight gain, loss of muscle tone, depression, fatigue, hair loss and, most importantly for many, erectile dysfunction and masculinity are key concerns. CNSs discuss and assess sexual function, providing prescriptions for PDE5 (phosphodiesterase 5) or MUSE (Medicated Urethral System for Erections), referring patients to pump clinics and running intercavernosal alprostadil injection clinics to teach patients how to inject into the corpora cavernosa to increase blood flow to the penis to produce an erections [11]. CNSs encourage all aspects of sexuality, including cuddling, mutual masturbation and oral sex or use of sex toys. CNSs are best placed to talk frankly about masculinity issues and identity. Body changes, such as breast enlargement or 'female emotionality', are adverse effects of endocrine manipulation. Bladder or bowel incontinence post-prostatectomy or radical radiotherapy could raise concerns about leaking urine during sexual intercourse leading to feeling 'less of a man!' A large meta-synthesis found that gay males felt they were unable to fulfil their sexual roles, expressing fear of rejection and stating that their partners would leave them [12]. Further data from this study found that older males appeared to accept and cope with therapy toxicity better than younger males with a prostate cancer diagnosis. CNSs are pivotal to alleviating fear and anxieties prostate cancer patients may experience post-therapy, and act as a key point of contact, advice and advocacy throughout the patient journey.

\section{Treatment Choices in the First-line Setting of Metastatic Hormone-Sensitive Prostate Cancer}

In recent years, several systemic therapies have been introduced into the advanced hormonesensitive prostate cancer space. ADT alone was for decades the standard of care in the treatment of these patients. This changed when studies demonstrated that the addition of docetaxel chemotherapy or abiraterone acetate to ADT significantly improves survival. This was followed by more recent evidence showing the efficacy of androgen receptor antagonists, such as enzalutamide and apalutamide. All four drugs have now been recommended for use in the metastatic hormone-sensitive prostate cancer (mHSPC) setting by the US FDA. The European Medicines Agency has also approved the use of docetaxel, abiraterone and apalutamide.

We have previously examined the relative efficacy of these four agents in a narrative review [13]. As of September 2020, there are no direct comparative trials between these agents, 
although most oncologists would agree that the efficacy data of the three novel anti-androgens and docetaxel chemotherapy are broadly comparable. In general, the novel anti-androgens have a more tolerable side-effect profile. In their respective clinical trials, the quality of life assessment demonstrated comparable or better outcomes than ADT monotherapy [14, 15]. Abiraterone demonstrated a superior quality of life to docetaxel within the STAMPEDE study up to 2 years of treatment, and particularly within the first year of treatment administration [16].

At the time of writing, oncologists in England and Wales are only reimbursed to prescribe docetaxel for mHSPC as treatment intensification. In the context of the COVID-19 pandemic, we are provisionally allowed to prescribe enzalutamide with ADT in the mHSPC setting, to 'reduce toxicity and potential for admission'. Patients who are intolerant to enzalutamide are permitted to switch to abiraterone, but only if it has been less than 3 months since enzalutamide was started.

The patient in this article has acquired abiraterone for mHSPC in the private sector, and we are pleased that he has demonstrated excellent response and tolerability to the drug and maintained a good quality of life after 3 years of treatment. He has been a key voice for patient awareness of prostate cancer and has been exemplary in keeping an active life despite treatment for advanced prostate cancer. He has also been strongly advocating the approval of abiraterone for use in other patients in the UK.

Significant developments and debate are occurring in this space. Earlier this year, NHS Scotland approved the use of abiraterone in the first-line mHSPC setting. Prostate Cancer UK and the British Uro-Oncology Group have just presented an appeal to NICE to reconsider their decision not to recommend the use of abiraterone in the mHSPC setting in England and Wales. Concurrently, updated data from the STAMPEDE trial presented at the ESMO (European Society for Medical Oncology) Congress 2020 in late September have demonstrated an impressive median overall survival of 6.6 years in the abiraterone cohort [17]. Despite the usual caveats of cross-trial comparisons, this is one of the most impressive efficacy results to date in this disease paradigm.
We acknowledge that the licensing and reimbursement of new treatments is a complex process, and for the purposes of this article, we will shy away from discussing health economics as it is beyond the remit of our expertise. However, as healthcare professionals, our primary duty of care is towards our patients. This duty encompasses ensuring that we balance the benefits and risks of all the treatments we offer our patients. As a profession, most of us would agree that there is a significant minority of patients who are ineligible for chemotherapy but would tolerate and benefit from the novel anti-androgen agents. There will also be patients who decline chemotherapy due to concerns about implications on work and lifestyle. Data from the National Prostate Cancer Audit 2020 have shown that only $27 \%$ of patients diagnosed with metastatic disease received docetaxel in England between April 2017 and March 2018 and that age, fitness and patient choice are significant factors in its uptake [18]. It is important to stress here that docetaxel is not without its relative merits. Docetaxel is delivered over 18 weeks in six outpatient infusions, compared to the novel antiandrogens which are taken up to progression. Most patients with a good level of physical fitness can tolerate this chemotherapeutic agent with few side effects. However, the option of administering novel anti-androgens in place of docetaxel will certainly expand the patient cohort who are able to receive treatment intensification, ensuring that a larger number of patients derive both survival and quality of life benefit.

Wherever and whenever possible, given comparable efficacy of treatments, we would advocate for shared decision-making with our patients about treatment choices. This would ensure that the treatment is tailored to the individual's co-morbidity profile, personal preferences and lifestyle.

\section{ACKNOWLEDGEMENTS}

Funding. No funding or sponsorship was received for this study or publication of this article. 
Authorship. The authors are fully responsible for all content. All named authors meet the International Committee of Medical Journal Editors (ICMJE) criteria for authorship for this article, take responsibility for the integrity of the work as a whole, and have given their approval for this version to be published. The opinions expressed in the manuscript are those of the authors.

Disclosures. Tony Collier, Shievon Smith and Michelle Greenwood have nothing to disclose. Kenrick Ng is currently receiving research funding from a Cancer Research UK Clinical Training Research Fellowship (Award Number 549580).

Compliance with Ethics Guidelines. This article does not contain any new studies with human or animal subjects performed by any of the authors.

Peer Review. Please note, contrary to the journal's standard single-blind peer review process, as a commentary this article underwent review by a member of the journal's Editorial Board.

Open Access. This article is licensed under a Creative Commons Attribution-NonCommercial 4.0 International License, which permits any non-commercial use, sharing, adaptation, distribution and reproduction in any medium or format, as long as you give appropriate credit to the original author(s) and the source, provide a link to the Creative Commons licence, and indicate if changes were made. The images or other third party material in this article are included in the article's Creative Commons licence, unless indicated otherwise in a credit line to the material. If material is not included in the article's Creative Commons licence and your intended use is not permitted by statutory regulation or exceeds the permitted use, you will need to obtain permission directly from the copyright holder. To view a copy of this licence, visit http://creativecommons.org/licenses/bync/4.0/.

\section{REFERENCES}

1. Hamdy FC, Donovan JL, Lane JA, et al. 10-Year Outcomes after monitoring, surgery, or radiotherapy for localized prostate cancer. $\mathrm{N}$ Engl J Med. 2016;375(15):1415-24.

2. Parekh DJ, Punnen S, Sjoberg DD, et al. A multiinstitutional prospective trial in the USA confirms that the 4Kscore accurately identifies men with high-grade prostate cancer. EurUrol. 2015;68(3): 464-70.

3. Ahmed HU, El-Shater Bosaily A, Brown LC, et al. Diagnostic accuracy of multi-parametric MRI and TRUS biopsy in prostate cancer (PROMIS): a paired validating confirmatory study. Lancet. 2017;389(10071):815-22.

4. Gronberg H, Adolfsson J, Aly M, et al. Prostate cancer screening in men aged 50-69 years (STHLM3): a prospective population-based diagnostic study. Lancet Oncol. 2015;16(16):1667-766.

5. Hodges CV. Studies on prostatic cancer I. the effect of castration, of estrogen and of androgen injection on serum phosphatases in metastatic carcinoma of the prostate. Cancer Res. 1941;1(4):293-7.

6. de Bono JS, De Giorgi U, Rodrigues DN, et al. Randomized phase II study evaluating Akt blockade with ipatasertib, in combination with abiraterone, in patients with metastatic prostate cancer with and without PTEN Loss. Clin Cancer Res. 2019;25(3): 928-36.

7. Bleyer A, Spreafico F, Barr R. Prostate cancer in young men: An emerging young adult and older adolescent challenge. Cancer. 2020;126(1):46-57.

8. Bracey M, Billing J, Turner C, Endacott R. Proving information early in the clinical pathway for people with prostate cancer. Cancer Nurs Pract. 2020;19:3.

9. Leary A, Brockson J, Endacott R, et al. The specialist nursing workforce caring for men with prostate cancer in the UK. Int J Urol Nurs. 2016;10(1):5-13.

10. Langelier DM, D'Silva A, Shank J, Grant C, Bridel W, Culos-Reed SN. Exercise interventions and their effect on masculinity, body image, and personal identity in prostate cancer-a systematic qualitative review. Psychooncology. 2019;28(6):1184-96.

11. Dinsmore WW, Wyllie MG. Vasoactive intestinal polypeptide/phentolamine for intracavernosal injection in erectile dysfunction. BJU Int. 2008;102(8):933-7.

12. Alexis O, Worsley AJ. A meta-synthesis of qualitative studies exploring men's sense of masculinity 
post-prostate cancer treatment. Cancer Nurs. 2018;41(4):298-310.

13. Ng K, Smith S, Shamash J. Metastatic hormonesensitive prostate Cancer (mHSPC): advances and treatment strategies in the first-line setting. Oncol Ther. 2020;15:1-22.

14. Chi KN, Agarwal N, Bjartell A, et al. Apalutamide for metastatic, castration-sensitive prostate cancer. N Engl J Med. 2019;381(1):13-24.

15. Chi KN, Protheroe A, Rodriguez-Antolin A, et al. Patient-reported outcomes following abiraterone acetate plus prednisone added to androgen deprivation therapy in patients with newly diagnosed metastatic castration-naive prostate cancer (LATITUDE): an international, randomised phase 3 trial. Lancet Oncol. 2018;19(2):194-206.
16. Rush HCA, Brawley C, Murphy L, Macnair A, Millman R. Comparative quality of life in patients randomized contemporaneously to docetaxel or abiraterone in the STAMPEDE trial. J Clin Oncol. 2020;38:6.

17. James DRH, Clarke N, Attard G, et al. Abiraterone acetate plus prednisolone for hormone-naive prostate cancer (PCa): Long-term results from metastatic (M1) patients in the STAMPEDE randomised trial (NCT00268476). Ann Oncol. 2020;31(4): S507-S549549.

18. National Prostate Cancer Audit (NPCA). Results of the NPCA Prospective Audit in England and Wales for men diagnosed from Apr 12017 to Mar 312018. 2020. https://www.npca.org.uk/content/uploads/ 2020/01/NPCA-Annual-Report-2019_090120.pdf. Accessed 20 Sept 2020. 